\title{
TOOLS
}

Science-Business eXchange

\section{Eau de fluorescence}

\section{By Joanne Kotz, Senior Editor}

Researchers from the National Cancer Institute and The University of Tokyo have developed a topical fluorescent probe that they have shown can detect residual tumor cells in mice during cancer surgery. ${ }^{1}$ The researchers will next validate the method in surgical tissue from ovarian cancer patients before submitting an IND.

The rationale for using fluorescent agents to tag tumor cells during cancer surgery is clear: they improve the efficiency of resection and allow better identification of residual cancer cells that could initiate metastases and might be missed by surgeons relying on the naked eye. The difficulty has been designing the probes.

Most agents in development are systemically delivered and have a lag time on the order of hours to days between when the agent is injected into the patient and when the fluorescent signal can be observed. Another challenge has been achieving a signal-to-noise ratio sufficient to distinguish tumor tissue from normal tissue.

A team led by Hisataka Kobayashi and Yasuteru Urano set out to develop a topical imaging agent that would quickly generate a strong fluorescent signal at tumor sites during surgery.

Kobayashi is chief scientist at NCI's Molecular Imaging Program. Urano is professor in the Laboratory of Chemical Biology and Molecular Imaging at the University of Tokyo.

The first step for the team was finding a tumor-specific enzyme that could be used to activate a topical fluorescent probe. They settled on $\gamma$-glutamyltransferase (GGT), which is overexpressed in multiple cancers including ovarian and cervical.

The team next synthesized the actual probe, $\gamma$-glutamyl hydroxymethyl rhodamine green (gGlu-HMRG). The probe was engineered to contain a molecular cage that quenches fluorescence. Once the probe comes in contact with the surface of a tumor cell, GGT cleaves the glutamate moiety off the probe, uncaging the probe and triggering a fluorescent signal.

In six xenograft mouse models of disseminated human ovarian cancer, which is characterized by the presence of small metastases throughout the peritoneal cavity, the researchers sprayed gGlu-HMRG onto the peritoneal surface with an endoscopic spray catheter, which is used clinically.

In four of the mice, the probe permitted visualization of tumors as small as $1 \mathrm{~mm}$ in diameter within minutes. The metastases were then readily removed with forceps using fluorescence-guided endoscopy.

In the other two mice, no fluorescence above background was observed, suggesting that not all ovarian cancers overexpress GGT.

Data were published in Science Translational Medicine.

\section{Make it fast}

The topical probe could be especially useful for procedures in which doctors want to visualize tumors on a limited and accessible surface.

"One significant advance of this imaging agent is the ability to visualize tumors within 10 minutes. This fast response opens up more real-time applications, which is exciting," said Tito Gonzalez, VP and head of R\&D at Avelas Biosciences Inc., which develops fluorescence approaches for intraoperative detection of tumors and metastases.

"Given the broad GGT expression and activity in blood, this technology will likely be restricted to topical applications," Gonzalez noted. "That being said, the technology is well suited for topical applications such as intraoperative identification of surface lesions," he added.

One possible application for the new probe during surgery could be spraying it on the remaining surface

"Given the broad GGT expression and activity in blood, this technology will likely be restricted to topical applications. That being said, the technology is well suited for topical applications such as intraoperative identification of surface lesions."

- Tito Gonzalez, Avelas Biosciences Inc. rumor, said Gooitzen van Dam, an associate professor and surgical oncologist at University Medical Center Groningen.

Instead of looking at the surgical surface after resection, researchers could also look at the resected tissue itself. "After taking tissue out of the patient, you could use the spray on the specimen to see if there is a positive margin" of normal tissue around the excised cancerous tissue, he added.

Van Dam was less convinced that a topical imaging agent would be useful for cancer surgeries in which it is necessary to assess distant lymph node metastases in addition to more localized spreading. As the lymph nodes are not accessible to a topical spray, he believes that a systemic agent would be necessary in these cases.

He added that the procedure may be particularly suited for repeated diagnostic monitoring of premalignant dysplasia for possible cancerous changes. For instance, he suggested that a topical fluorescent agent could be used to look for malignancies during routine dermatology visits.

\section{Spraying power}

The potential limitations of the new probe include tissue penetration, inactivation by an inflammation response and a potentially low signalto-noise ratio.

"Spraying raises questions as to the homogeneity of the administration and penetration depth. For spraying approaches, diffusion into the tissue is not well controlled," said Vasilis Ntziachristos, director of the Institute for Biological and Medical Imaging at the German Research Center for Environmental Health and chair for biological and medical imaging at the Technical University Munich.

"I am very interested in how this will behave if you have much more overlying tissue-will it penetrate well? Also, will it be hampered by 


\section{ANALYSIS}

an inflammatory response [in] surrounding tissue?" asked van Dam. He added that experiments using human tissue should address his questions.

"One big question will be how GGT activity in human tumors compares to GGT expression in noncancerous human tissue. Hopefully, the background will be as low as has been shown for the mouse," said Gonzalez.

"A challenge of this approach is how variable enzyme expression levels in different cancer lines result in different fluorescence responses. I suspect that in less homogenous models the ability to separate cancer from GGT-expressing background tissue will be more challenging," noted Gonzalez.

The team's next step will be to evaluate the probe in tissue from ovarian cancer patients.

"We have prepared a clinical protocol for evaluating the efficacy of this probe using fresh surgical ovarian cancer specimens in the surgery room. After this validation, we will prepare and apply for an IND," said Kobayashi.

The researchers also are exploring the applicability of the probe to other cancers, including cervical, uterine, gastric, hepatic and colon.
Urano told SciBX that the University of Tokyo has filed for two patents covering the work. The IP is available for licensing.

Kotz, J. SciBX 5(1); doi:10.1038/scibx.2012.4

Published online Jan. 5, 2012

\section{REFERENCES}

1. Urano, Y. et al. Sci. Transl. Med.; published online Nov. 23, 2011; doi:10.1126/scitransImed.3002823

Contact: Hisataka Kobayashi, National Institutes of Health, Bethesda, Md.

e-mail: kobayash@mail.nih.gov

Contact: Yasuteru Urano, The University of Tokyo, Tokyo, Japan e-mail: uranokun@m.u-tokyo.ac.jp

COMPANIES AND INSTITUTIONS MENTIONED

Avelas Biosciences Inc., San Diego, Calif.

German Research Center for Environmental Health, Munich, Germany

National Cancer Institute, Bethesda, Md.

Technical University Munich, Munich, Germany

University Medical Center Groningen, Groningen, the

Netherlands

The University of Tokyo, Tokyo, Japan 